\title{
INCOMPRESSIBLE EULER AND E-MHD AS SCALING LIMITS OF THE VLASOV-MAXWELL SYSTEM*
}

\author{
YANN BRENIER ${ }^{\dagger}$, NORBERT MAUSER $^{\ddagger}$, AND MARJOLAINE PUEL ${ }^{\S}$
}

\begin{abstract}
We consider two different asymptotic limits of the Vlasov-Maxwell system describing a quasineutral plasma with a uniform ionic background. In the first case, as the magnetic field is preserved in the limiting process, we obtain the so-called electron magnetohydrodynamics equations. In the second case, we obtain the incompressible Euler equations with no more magnetic field left.
\end{abstract}

\section{Introduction}

Let us consider the Vlasov-Maxwell system

$$
\begin{gathered}
\partial_{t} f+\xi \cdot \nabla_{x} f-(E+\alpha(\xi \wedge B)) \cdot \nabla_{\xi} f=0, \\
\alpha \partial_{t} B+\nabla \wedge E=0 \quad, \quad \varepsilon \nabla \cdot E=1-\rho, \\
\varepsilon \alpha \partial_{t} E-\nabla \wedge B=\alpha J, \quad \nabla \cdot B=0,
\end{gathered}
$$

This system (1.1)-(1.3) describes the evolution of the electron phase space density $f(t, x, \xi) \geq 0$ at time $t>0$ and point $x \in \mathbb{R}^{3}$, with velocity $\xi \in \mathbb{R}^{3}$, in a uniform background of non-moving ions with unit density. The fields $E$ and $B$ are respectively the electric field and the magnetic field. The density $\rho$ and the current $J$ are given by

$$
\rho=\int f(t, x, \xi) d \xi \quad, \quad J=\int \xi f(t, x, \xi) d \xi .
$$

$E$ and $B$ are coupled to the electron density through the Maxwell equations and act on electrons via the Lorentz force. Notice that we do not consider here the relativistic Vlasov-Maxwell system for which the impulse variable $\xi$ and the velocity variable are not proportional. (This means that the speed of light must be infinite at leading order in any reasonable scaling.) After non-dimensionalization, parameters $\alpha$ and $\epsilon$ can be chosen independently of each other, according to the desired scaling. Indeed, in physical units

$$
\alpha=\sqrt{\frac{r_{0}}{\varepsilon_{0}}}, \quad \epsilon=\frac{\varepsilon_{0}}{r_{0} c^{2}},
$$

where $r_{0}$ is the classical electron radius $\left(r_{0}=2.82 \times 10^{-15} \mathrm{~m}\right), \varepsilon_{0}$ is the vacuum dielectric constant and $c$ is the speed of light.

We will concentrate on the so-called quasi-neutral regime when $\epsilon$ is a small parameter. Taking the first two moments of (1.1), we obtain the following system,

$$
\begin{array}{r}
\partial_{t} \rho+\nabla \cdot J=0 \\
\partial_{t} J+\nabla \cdot\left(\int \xi \otimes \xi f d \xi\right)+E \rho+\alpha J \wedge B=0
\end{array}
$$

*Received: April 10, 2003; accepted (in revised version): May 24, 2003. Communicated by Francois Golse.

${ }^{\dagger}$ CNRS, UMR 6621, Parc Valrose, F-06108 cedex 2, Nice, France (brenier@ann.jussieu.fr).

${ }^{\ddagger}$ Wolfgang Pauli Institute c/o Inst. f. Mathematik Strudlhofg. 4, A-1090 Wien, Austria (norbert.mauser@univie.ac.at).

${ }^{\S}$ CEREMADE Place du Marchal de Lattre de TASSIGNY, F-75775 Paris, Cedex 16, France (mpuel@ceremade.dauphine.fr). 
REMARK 1.1. Here the notation $\nabla \cdot\left(\int \xi \otimes \xi f d \xi\right)$ corresponds to

$$
\sum_{j=1}^{3} \partial_{j} \int \xi_{i} \xi_{j} f d \xi, \quad i=1,2,3 .
$$

Starting from this system, we may consider two different quasi-neutral scalings.

Scaling 1: $\alpha<<1, \varepsilon<<1$

This scaling corresponds to a nonrelativistic limit, where the magnetic field vanishes, coupled with a quasi-neutral limit (the electron density instantaneously adjusts itself to the unit background density). Formally, it is easy to guess the limit of (1.5),(1.6), (1.2),(1.3) when $\varepsilon, \alpha \rightarrow 0$, provided that the distribution function tends to be monokinetic, i.e.

$$
f(t, x, \xi) \sim \rho(t, x) \delta\left(\xi-\frac{J(t, x)}{\rho(t, x)}\right),
$$

which means that the electronic temperature is close to zero and implies

$$
\int \xi \otimes \xi f d \xi \sim \frac{J \otimes J}{\rho} .
$$

This limit is the Euler equation of incompressible fluids

$$
\begin{aligned}
& \partial_{t} J+\nabla \cdot(J \otimes J)+E=0, \\
& \nabla \cdot J=0 \quad, \quad \rho=1, \\
& \nabla \wedge E=0, \quad B=0 .
\end{aligned}
$$

Scaling 2: $\alpha=1, \varepsilon<<1$

Now, the magnetic field does not disappear any longer in the limit equations, the so-called electron magnetohydrodynamics equations $[\mathrm{KCY}]$ :

$$
\begin{array}{r}
\partial_{t} J+\nabla \cdot(J \otimes J)+E+J \wedge B=0, \\
J=-\nabla \wedge B \quad, \quad \rho=1, \\
\partial_{t} B+\nabla \wedge E=0, \quad \nabla \cdot B=0 .
\end{array}
$$

As above, provided that the distribution function tends to be monokinetic, the formal limit is obvious.

In the present paper, we provide a rigorous derivation of these formal limits by using the modulated energy method designed in $[\mathrm{Br}]$ for the quasi-neutral limit of the Vlasov-Poisson system. This method has been used in a quantum context in [Pu1], [Pu2] based on the concept of dissipative solutions due to P.-L. Lions [Li]. This method can be seen as a variant of both the relative entropy method [Da], [Ya] and the Hamiltonian energy method by E. Grenier [Gr].

The key idea is to estimate a modulation of the energy by the solution of the formal limit equation. Typically, we replace (twice) the kinetic energy $\int|\xi|^{2} f d \xi$ by $\int|\xi-v|^{2} f d \xi$ where $v$ is a smooth solution to the limit equations.

The Vlasov-Maxwell system is known to admit global strong solutions at least for smooth initial data depending only on two space coordinates, with a perpendicular magnetic field (see [GlSc] for details and [DiLi] for global weak solutions). To keep the proofs as simple as possible, we state our results in the case of spatially periodic solutions on the unit cube. All spatial integrals will be implicitly performed on the unit cube. 


\section{Convergence to the electron-Magnetohydrodynamics (e-MHD)}

2.1. Result. In this section, we consider the Vlasov-Maxwell system (1.1), $(1.2),(1.3),(1.4)$, where $\alpha>0$ is a fixed constant of order one and $\varepsilon$ is a small parameter. (This means that the magnetic field will not vanish in the limiting process.) The system that we consider for the limit $\varepsilon \rightarrow 0$, is the so-called e-MHD system [KCY]

$$
\begin{aligned}
& \partial_{t} v+(v \cdot \nabla) v+e+\alpha v \wedge b=0 \\
& \text { with } \alpha \partial_{t} b+\nabla \wedge e=0, \alpha v=\nabla \wedge b \text { and } \nabla \cdot b=0 .
\end{aligned}
$$

Introducing $\omega=\nabla \wedge(v-\alpha A)$ where $A$ is the magnetic potential such that $\nabla \wedge A=b$ and $\nabla \cdot A=0$, and using the identity

$$
(v \cdot \nabla) v=(\nabla \wedge v) \wedge v+\nabla \frac{|v|^{2}}{2},
$$

we can write e-MHD in a different way :

$$
\partial_{t} \omega+v \cdot \nabla \omega=\omega \cdot \nabla v, \text { with }-\Delta v+\alpha^{2} v=\nabla \wedge \omega .
$$

In particular, as the initial data depend only on the two first coordinates $x_{1}, x_{2}$, then $\omega$ is aligned with the third coordinate and can be seen as a scalar field satisfying

$$
\partial_{t} \omega+v \cdot \nabla \omega=0, \text { with }-\Delta v+\alpha^{2} v={ }^{\perp} \nabla \omega=\left(-\partial_{1} \omega_{2}, \partial_{2} \omega_{1}, 0\right),
$$

Therefore, the existence results are the same as for the incompressible Euler equations (see [Li], for instance). In particular, we have global strong solutions for smooth initial conditions depending only on two space coordinates. Let us now state our first main result

Theorem 2.1. Let us fix $T>0, \alpha>0$ and $C>0$. Let $(f, B, E)$ and $(v, b, e)$ be two smooth solutions of respectively the VM system and the e-MHD system on the time interval $[0, T]$. Assume their initial values to satisfy

$$
\begin{gathered}
\int f(0, x, \xi) d x d \xi=1, \\
\int f(0, x, \xi)|\xi|^{2} d x d \xi \leq C, \\
\varepsilon \int|E(0, x)|^{2} d x \leq C \sqrt{\varepsilon}, \\
\int|B(0, x)-b(0, x)|^{2} d x \leq C \sqrt{\varepsilon}, \\
\int|\xi-v(0, x)|^{2} f(0, x, \xi) d x d \xi \leq C \sqrt{\varepsilon} .
\end{gathered}
$$

Then

$$
\sup _{0 \leq t \leq T}\left(\int|\xi-v(t, x)|^{2} f(t, x, \xi) d \xi d x+\int|B(t, x)-b(t, x)|^{2} d x\right) \leq C^{\prime} \sqrt{\epsilon},
$$

where $C^{\prime}$ depends only on $T, C$ and $(v, b, e)$. 
2.2. Moment equations and modulated energy. We deduce from the Vlasov system the following equations for the moments $\rho$ and $J$ :

$$
\partial_{t} \rho+\nabla \cdot J=0
$$

and

$$
\partial_{t} J+\nabla \cdot\left(\int \xi \otimes \xi f d \xi\right)+\rho E+\alpha J \wedge B=0
$$

and the conserved energy is

$$
H=\frac{1}{2} \int|\xi|^{2} f(t, x, \xi) d \xi d x+\int \frac{\varepsilon|E(t, x)|^{2}+|B(t, x)|^{2}}{2} d x .
$$

Following $[\mathrm{Br}]$, we define a 'modulated energy' $H_{v, b}(t)$ by

$$
H_{v, b}(t):=\frac{1}{2} \int|\xi-v(t, x)|^{2} f(t, x, \xi) d \xi d x+\int \frac{\varepsilon|E(t, x)|^{2}+|B(t, x)-b(t, x)|^{2}}{2} d x
$$

where $v$ and $b$ are arbitrarily chosen smooth divergence free vector fields depending on $t \geq 0$ and $x \in \mathbb{R}^{3} / \mathbb{Z}^{3}$.

Proposition 2.2. Let $(f, B, E)$ be a smooth solution to the VM system and $v, b, e$ three smooth vector fields, $b$ and $v$ being divergence free, then the modulated energy $H_{v, b}$ satisfies

$$
\frac{d}{d t}\left(H_{v, b}-\theta\right)=\int \sum_{i, j=1}^{3} \partial_{j} v_{i}\left[-\int(\xi-v)_{i}(\xi-v)_{j} f d \xi+(B-b)_{i}(B-b)_{j}+\varepsilon E_{i} E_{j}\right] d x+\eta+r
$$

where

$$
\begin{gathered}
\theta=\varepsilon \int E \cdot(e+\alpha v \wedge(b-B)) d x \\
\eta=-\varepsilon \int E \cdot\left(\partial_{t} e+\nabla(e \cdot v)+\alpha \partial_{t}(v \wedge b)-\alpha \partial_{t} v \wedge B\right) d x,
\end{gathered}
$$

and

$$
\begin{gathered}
r=-\int(e-E-\alpha v \wedge B) \cdot\left(\frac{\nabla \wedge b}{\alpha}+v\right) d x \\
-\int(B-b) \cdot\left(\partial_{t} b+\frac{\nabla \wedge e}{\alpha}\right) d x-\int\left(\left(\partial_{t}+v \cdot \nabla\right) v+e+\alpha v \wedge b\right) \cdot(J-\rho v) d x .
\end{gathered}
$$

Theorem 2.1 follows easily from Proposition 2.2. Indeed, we first assume $(v, b, e)$ to be solution to the e-MHD equation. As a consequence, $r$ vanishes in the right-hand side of (2.8). Next, it follows from the assumptions of Theorem 2.1 that $\eta(t)$ and $\theta(t)$ are uniformly bounded by $C_{1} \sqrt{\varepsilon}$, for $t \in[0, T]$ where $C_{1}$ depends only on $T, C$ and $(v, b, e)$. Thus, we get from (2.8),

$$
\frac{d}{d t}\left(H_{v, b}-\theta\right) \leq 2 \lambda H_{v, b}+\eta \leq 2 \lambda\left(H_{v, b}-\theta\right)+2 \lambda \theta+\eta \leq 2 \lambda\left(H_{v, b}-\theta+C_{2} \sqrt{\varepsilon}\right),
$$


where

$$
\lambda=\sup _{t, x, \xi} \sum_{i, j=1}^{3} \partial_{j} v_{i}(t, x) \frac{\xi_{i} \xi_{j}}{|\xi|^{2}}
$$

and $C_{2}$ depends only on $T, C$ and $(v, b, e)$. Thus

$$
H_{v, b}(t)-\theta(t)+C_{2} \sqrt{\varepsilon} \leq \exp (2 \lambda t)\left(H_{v, b}(0)-\theta(0)+C_{2} \sqrt{\varepsilon}\right) .
$$

From the assumptions of Theorem 2.1, we know that $H_{v, b}(0) \leq 1.5 C \sqrt{\varepsilon}$ and conclude that

$$
H_{v, b}(t) \leq C^{\prime} \sqrt{\varepsilon}
$$

where $C^{\prime}$ depends only on $T, C$ and $(v, b, e)$ and the result of Theorem 2.1 easily follows.

2.3. Proof of Proposition 2.2. N.B. To perform the calculations required by the proof, we use notations with indices $i, j, k \in\{1,2,3\}$, implicit summation on repeated indices and abridged notations with comma for partial derivatives. For instance, $v_{, t}+v_{i, j} v_{j}$ stands for $\partial_{t} v+(v \cdot \nabla) v$. Consistently, we use the signature symbol $\epsilon_{i j k}=+1,-1$ to denote the wedge product and the curl operator. With these notations, the equations satisfied by $(\rho, J, E, B)$ are

$$
\begin{gathered}
\rho_{, t}=-J_{j, j}, \\
-J_{i, t}=\left(\int \xi_{i} \xi_{j} f d \xi\right)_{, j}+\alpha \epsilon_{i j k} J_{j} B_{k}+\rho E_{i}, \\
\alpha B_{i, t}=-\epsilon_{i j k} E_{k, j}, \quad B_{i, i}=0, \\
\alpha J_{i}=\varepsilon \alpha E_{i, t}-\epsilon_{i j k} B_{k, j}, \quad \rho=1-\varepsilon E_{i, i} .
\end{gathered}
$$

Let us notice that we can write equation (2.10) in a different way, using equations (2.11) and (2.12), namely :

$$
\begin{gathered}
-J_{i, t}=\left(\int \xi_{i} \xi_{j} f d \xi\right)_{, j}-\epsilon_{i j k} \epsilon_{j p q} B_{q, p} B_{k}+\varepsilon \alpha \epsilon_{i j k} E_{j, t} B_{k}+E_{i}\left(1-\varepsilon E_{j, j}\right) \\
=\left(\int \xi_{i} \xi_{j} f d \xi\right)_{, j}+B_{k} B_{k, i}-B_{k} B_{i, k}+\varepsilon \alpha \epsilon_{i j k}\left(E_{j} B_{k}\right)_{, t}+\varepsilon \alpha \epsilon_{i j k} E_{j} B_{k, t}+E_{i}\left(1-\varepsilon E_{j, j}\right) \\
=\left(\int \xi_{i} \xi_{j} f d \xi\right)_{, j}+B_{j}\left(B_{j, i}-B_{i, j}\right)+\varepsilon \alpha \epsilon_{i j k}\left(E_{j} B_{k}\right)_{, t}+\varepsilon \epsilon_{i j k} \epsilon_{k p q} E_{j} E_{q, p}+E_{i}\left(1-\varepsilon E_{j, j}\right)
\end{gathered}
$$

(using (2.11). Thus,

$-J_{i, t}=\left(\int \xi_{i} \xi_{j} f d \xi\right)_{, j}+B_{j}\left(B_{j, i}-B_{i, j}\right)+\varepsilon \alpha \epsilon_{i j k}\left(E_{j} B_{k}\right)_{, t}+\varepsilon E_{j}\left(E_{j, i}-E_{i, j}\right)+E_{i}\left(1-\varepsilon E_{j, j}\right)$. 
The modulated energy is defined by

$$
H_{b, v}=\frac{1}{2}\left(\iint|\xi-v|^{2} f d \xi+\int|B-b|^{2}+\int \varepsilon|E|^{2}\right) .
$$

We have

$$
H_{b, v}=H+\int\left(-J_{i} v_{i}+\rho v_{i} v_{i} / 2\right)+\int\left(-B_{i} b_{i}+b_{i} b_{i} / 2\right)
$$

where the energy

$$
H=H_{0,0}=\frac{1}{2}\left(\iint|\xi|^{2} f d \xi+\int|B|^{2}+\int \varepsilon|E|^{2}\right)
$$

is conserved and therefore does not depend on $t$. Thus

$$
\frac{d H_{b, v}}{d t}=Q_{1}+Q_{2}+Q_{3}+L_{1}+L_{2}+L_{3},
$$

where

$$
\begin{gathered}
Q_{1}=-\int v_{i} J_{i, t}, \quad Q_{2}=\int \rho_{, t} v_{i} v_{i} / 2, \quad Q_{3}=-\int B_{i, t} b_{i}, \\
L_{1}=-\int J_{i} v_{i, t}, \quad L_{2}=\int \rho v_{i} v_{i, t}, \quad L_{3}=-\int(B-b)_{i} b_{i, t} .
\end{gathered}
$$

From equation (2.13), we deduce

$$
\begin{gathered}
Q_{1}=\int v_{i}\left(\int \xi_{i} \xi_{j} f d \xi\right)_{, j}+\int v_{i} B_{j}\left(B_{j, i}-B_{i, j}\right)+\varepsilon \alpha \int \epsilon_{i j k} v_{i}\left(E_{j} B_{k}\right)_{, t} \\
+\varepsilon \int v_{i} E_{j}\left(E_{j, i}-E_{i, j}\right)+\int v_{i} E_{i}\left(1-\varepsilon E_{j, j}\right) \\
=\int v_{i}\left(\int \xi_{i} \xi_{j} f d \xi\right)_{, j}+\int v_{i}\left(\left(\frac{|B|^{2}}{2}\right)_{, i}-B_{j} B_{i, j}\right)+\varepsilon \alpha \int \epsilon_{i j k} v_{i}\left(E_{j} B_{k}\right)_{, t} \\
+\varepsilon \int v_{i}\left(\left(\frac{|E|^{2}}{2}\right)_{, i}-\left(E_{i} E_{j}\right)_{, j}\right)+\int v_{i} E_{i} .
\end{gathered}
$$

Thus, after integrating by part and using that $v_{j, j}=B_{j, j}=0$,

$$
Q_{1}=\int v_{i, j}\left(-\int \xi_{i} \xi_{j} f d \xi+B_{j} B_{i}+\varepsilon E_{j} E_{i}\right)+\int v_{i} E_{i}+\eta_{0}+\frac{d}{d t} \theta_{0},
$$

where

$$
\theta_{0}=\varepsilon \alpha \int \epsilon_{i j k} v_{i} E_{j} B_{k}, \quad \eta_{0}=-\varepsilon \alpha \int \epsilon_{i j k} v_{i, t} E_{j} B_{k}
$$


Next, from equation (2.9),

$$
Q_{2}=\int \rho_{, t} v_{i} v_{i} / 2=-\int J_{j, j} v_{i} v_{i} / 2=\int J_{j} v_{i} v_{i, j}
$$

and, from (2.11),

$$
Q_{3}=-\int B_{k, t} b_{k}=\int \epsilon_{k j i} \frac{E_{i, j}}{\alpha} b_{k}=\int E_{i} \epsilon_{i j k} \frac{b_{k, j}}{\alpha} .
$$

So

$$
\begin{gathered}
Q=Q_{1}+Q_{2}+Q_{3}=\int v_{i, j}\left(-\int \xi_{i} \xi_{j} f d \xi+B_{i} B_{j}+\varepsilon E_{i} E_{j}\right)+\int J_{j} v_{i} v_{i, j}+\eta_{0}+\frac{d}{d t} \theta_{0} \\
+r_{0}
\end{gathered}
$$

where

$$
r_{0}=\int E_{i}\left(\epsilon_{i j k} \frac{b_{k, j}}{\alpha}+v_{i}\right) .
$$

(Notice that $r_{0}=0$ as $(b, v, e)$ satisfies the e-MHD system.) We also have

$$
L_{3}=-\int(B-b)_{i} b_{i, t}=\int(B-b)_{i} \frac{\epsilon_{i j k} e_{k, j}}{\alpha}+r_{1},
$$

where

$$
r_{1}=-\int(B-b)_{i}\left(b_{i, t}+\frac{\epsilon_{i j k} e_{k, j}}{\alpha}\right) .
$$

(Notice that $r_{1}=0$ as $(b, v, e)$ satisfies the e-MHD system.) Thus

$$
\begin{gathered}
L_{3}=-\int e_{k} \epsilon_{i j k} \frac{(B-b)_{i, j}}{\alpha}+r_{1} \\
=-\int e_{k}\left(J_{k}-\varepsilon E_{k, t}-v_{k}\right)+r_{1}+r_{2},
\end{gathered}
$$

(because of equation (2.12)), where

$$
r_{2}=-\int e_{k}\left(v_{k}+\frac{\epsilon_{k j i} b_{i, j}}{\alpha}\right)
$$

(Notice that $r_{2}=0$ if $(b, v, e)$ is solution to the e-MHD system.) So

$$
\begin{gathered}
L_{3}=-\int e_{k}\left(J_{k}-\rho v_{k}\right)-\int e_{k}(\rho-1) v_{k}+\frac{d}{d t} \varepsilon \int E_{k} e_{k}-\varepsilon \int E_{k} e_{k, t}+r_{1}+r_{2} \\
=-\int e_{k}\left(J_{k}-\rho v_{k}\right)+\eta_{1}+\eta_{2}+\frac{d}{d t} \theta_{1}+r_{1}+r_{2}
\end{gathered}
$$


(using $\left.\rho=1-\varepsilon E_{i, i}\right)$ where

$$
\begin{gathered}
\eta_{1}=-\varepsilon \int\left(e_{k} v_{k}\right)_{, i} E_{i}, \\
\eta_{2}=-\varepsilon \int E_{k} e_{k, t} \\
\theta_{1}=\varepsilon \int E_{k} e_{k} .
\end{gathered}
$$

It follows, using definition (2.17), that

$$
L=L_{1}+L_{2}+L_{3}=-\int\left(v_{k, t}+e_{k}\right)\left(J_{k}-\rho v_{k}\right)+\eta_{1}+\eta_{2}+\frac{d}{d t} \theta_{1}+r_{1}+r_{2}
$$

Thus

$$
L=\int v_{i, j}\left(v_{j} J_{i}-\rho v_{j} v_{i}\right)+L_{4}+\eta_{1}+\eta_{2}+\frac{d}{d t} \theta_{1}+r_{1}+r_{2}+r_{3}
$$

where

$$
r_{3}=-\int\left(v_{i, t}+v_{j} v_{i, j}+e_{i}+\alpha \epsilon_{i j k} v_{j} b_{k}\right)\left(J_{i}-\rho v_{i}\right)
$$

(which vanishes if $(b, v, e)$ is a solution to the e-MHD system), and

$$
\begin{gathered}
L_{4}=\int \alpha \epsilon_{i j k} v_{j} b_{k}\left(J_{i}-\rho v_{i}\right) \\
=\int \alpha \epsilon_{i j k} v_{j} b_{k} J_{i}=\varepsilon \alpha \int \epsilon_{i j k} v_{j} b_{k} E_{i, t}-\int \epsilon_{i j k} v_{j} b_{k} \epsilon_{i p q} B_{q, p}
\end{gathered}
$$

(because of (2.12))

$$
=\eta_{3}+\frac{d}{d t} \theta_{2}+L_{5}
$$

where

$$
\begin{gathered}
\eta_{3}=-\varepsilon \alpha \int \epsilon_{i j k}\left(v_{j} b_{k}\right)_{, t} E_{i}, \\
\theta_{2}=\varepsilon \alpha \int \epsilon_{i j k} v_{j} b_{k} E_{i}, \\
L_{5}=-\int v_{j} b_{k}\left(B_{k, j}-B_{j, k}\right) .
\end{gathered}
$$

We have

$$
L_{5}=\int v_{j} b_{k, j} B_{k}-\int v_{j, k} b_{k} B_{j}
$$


(since both $v$ and $b$ are divergence free)

$$
\begin{gathered}
=-\int v_{j, k}\left(b_{k} B_{j}+B_{k} b_{j}\right)+\int v_{j}\left(b_{k, j}-b_{j, k}\right) B_{k} \\
=-\int v_{j, k}\left(b_{k} B_{j}+B_{k} b_{j}\right)+\int \epsilon_{i j k} \epsilon_{i p q} b_{q, p} v_{j} B_{k} \\
=-\int v_{j, k}\left(b_{k} B_{j}+B_{k} b_{j}\right)+r_{4},
\end{gathered}
$$

where

$$
r_{4}=\int \epsilon_{i j k}\left(\epsilon_{i p q} b_{q, p}+\alpha v_{i}\right) v_{j} B_{k}
$$

(and vanishes if $(b, v, e)$ is solution to the e-MHD system). So

$$
L_{4}=\eta_{3}+\frac{d}{d t} \theta_{2}-\int v_{j, k}\left(b_{k} B_{j}+B_{k} b_{j}\right)+r_{4}
$$

and

$$
\begin{gathered}
L=\int v_{i, j}\left(v_{j} J_{i}-\rho v_{j} v_{i}-b_{i} B_{j}-B_{i} b_{j}\right) \\
+\eta_{1}+\eta_{2}+\eta_{3}+\frac{d}{d t}\left(\theta_{1}+\theta_{2}\right)+r_{1}+r_{2}+r_{3}+r_{4}
\end{gathered}
$$

It follows, using definition (2.16), that

$$
L+Q=Q^{\prime}+\eta_{0}+\eta_{1}+\eta_{2}+\eta_{3}+\frac{d}{d t}\left(\theta_{0}+\theta_{1}+\theta_{2}\right)+r_{0}+r_{1}+r_{2}+r_{3}+r_{4},
$$

where

$$
Q^{\prime}=\int v_{i, j}\left((B-b)_{j}(B-b)_{i}+\varepsilon E_{j} E_{i}-\int\left(\xi_{i}-v_{i}\right)\left(\xi_{j}-v_{j}\right) f d \xi\right) .
$$

So, we have finally obtained

$$
\frac{d}{d t}\left(H_{b, v}-\theta\right)=Q^{\prime}+\eta+r
$$

where

$$
\begin{gathered}
\eta=\eta_{0}+\eta_{1}+\eta_{2}+\eta_{3}=-\varepsilon \int \epsilon_{i j k} \alpha v_{i, t} E_{j} B_{k}-\varepsilon \int E_{i}\left(e_{i, t}+\left(e_{k} v_{k}\right)_{, i}+\alpha \epsilon_{i j k}\left(v_{j} b_{k}\right), t\right), \\
\theta=\theta_{0}+\theta_{1}+\theta_{2}=\varepsilon \alpha \int \epsilon_{i j k} v_{i} E_{j} B_{k},+\varepsilon \int E_{i}\left(e_{i}+\alpha \epsilon_{i j k} v_{j} b_{k}\right)
\end{gathered}
$$

and

$$
r=r_{0}+r_{1}+r_{2}+r_{3}+r_{4}=\int E_{i}\left(\epsilon_{i j k} \frac{b_{k, j}}{\alpha}+v_{i}\right)-\int(B-b)_{i}\left(b_{i, t}+\frac{\epsilon_{i j k} e_{k, j}}{\alpha}\right)
$$




$$
\begin{gathered}
-\int e_{k}\left(v_{k}+\frac{\epsilon_{k j i} b_{i, j}}{\alpha}\right)-\int\left(v_{i, t}+v_{j} v_{i, j}+e_{i}+\alpha \epsilon_{i j k} v_{j} b_{k}\right)\left(J_{i}-\rho v_{i}\right) \\
+\int \epsilon_{i j k}\left(\epsilon_{i p q} b_{q, p}+\alpha v_{i}\right) v_{j} B_{k} \\
=-\int\left(e_{i}-E_{i}-\epsilon_{i p q} \alpha v_{p} B_{q}\right)\left(\epsilon_{i j k} \frac{b_{k, j}}{\alpha}+v_{i}\right) \\
-\int(B-b)_{i}\left(b_{i, t}+\frac{\epsilon_{i j k} e_{k, j}}{\alpha}\right)-\int\left(v_{i, t}+v_{j} v_{i, j}+e_{i}+\alpha \epsilon_{i j k} v_{j} b_{k}\right)\left(J_{i}-\rho v_{i}\right) .
\end{gathered}
$$

This completes the proof of Proposition 2.2.

\section{Convergence to the Euler equation}

3.1. Result. In this section, we consider the Vlasov-Maxwell system (1.1), (1.2), (1.3), (1.4), with a different scaling where both $\alpha$ and $\varepsilon$ go to zero. The limit system is now the usual Euler equations for incompressible fluids

$$
\begin{aligned}
& \partial_{t} v+(v \cdot \nabla) v+e=0 \\
& \text { with } \nabla \wedge e=0, \text { and } \nabla \cdot v=0 .
\end{aligned}
$$

Let us recall that, by introducing $\omega=\nabla \wedge v$, we get the standard vorticity formulation of the Euler equations, namely :

$$
\partial_{t} \omega+v \cdot \nabla \omega=\omega \cdot \nabla v, \text { with }-\Delta v=\nabla \wedge \omega .
$$

Let us now state our second main result

Theorem 3.1. Let us fix $T>0$ and $C>0$. Let $(f, B, E)$ and $(v, e)$ be two smooth solutions of respectively the VM system and the Euler system on the time interval $[0, T]$. For each $t \in[0, T]$, assume $v$ to have zero mean and introduce $\beta$ such that

$$
\nabla \wedge \beta=v, \quad \nabla \cdot \beta=0 .
$$

Assume the initial values to satisfy

$$
\begin{gathered}
\int f(0, x, \xi) d x d \xi=1 \\
\int f(0, x, \xi)|\xi|^{2} d x d \xi \leq C \\
\varepsilon \int|E(0, x)|^{2} d x \leq(\alpha+\sqrt{\varepsilon}) C \\
\int|B(0, x)-\alpha \beta(0, x)|^{2} d x \leq(\alpha+\sqrt{\varepsilon}) C \\
\int|\xi-v(0, x)|^{2} f(0, x, \xi) d x d \xi \leq(\alpha+\sqrt{\varepsilon}) C .
\end{gathered}
$$

Then

$$
\sup _{0 \leq t \leq T}\left(\int|\xi-v(t, x)|^{2} f(t, x, \xi) d \xi d x+\int|B(t, x)-\alpha \beta(t, x)|^{2} d x\right) \leq(\alpha+\sqrt{\epsilon}) C^{\prime}
$$

where $C^{\prime}$ depends only on $T, C$ and $(v, e)$. 
3.2. Proof of Theorem 3.1. We use again Proposition 2.2, but with a different choice for $(v, b, e)$. We assume $(v, e)$ to be the smooth solution of the Euler equation considered in the assumptions of Theorem 3.1, and define

$$
b(t, x)=\alpha \beta(t, x), \quad \nabla \wedge \beta=v, \quad \nabla \cdot \beta=0 .
$$

This implies that

$$
\begin{gathered}
\theta=\varepsilon \int E \cdot(e+\alpha v \wedge(\alpha \beta-B)) \\
\eta=-\varepsilon \int E \cdot\left(\partial_{t} e+\nabla(e \cdot v)+\alpha^{2} \partial_{t}(v \wedge \beta)-\alpha \partial_{t} v \wedge B\right),
\end{gathered}
$$

and

$$
r=-\int(B-\alpha \beta) \cdot \alpha \partial_{t} \beta-\int \alpha^{2} v \wedge \beta \cdot(J-\rho v) .
$$

(using that $\nabla \wedge \beta=v$ and $(v, e)$ is a solution to the Euler equations which in particular implies $\nabla \wedge e=0)$. Thus, $\eta, \theta$ and $r$ are uniformly bounded by $(\alpha+\sqrt{\epsilon}) C^{\prime \prime}$ for some constant $C^{\prime \prime}$ depending only on $T,(b, v)$ and the total energy $H$. Then, the proof of Theorem 3.1 immediately follows as in the previous section for Theorem 2.1.

Acknowledgments. The authors acknowledge the support from the European IHP network "HYKE" HPRN-CT-2002-00282, the LRC CEA-Cadarache/UNSA, the START project (FWF, TEC-Y-137) of N.J.M and the Austrian-French program "Amadeus". Y.B. thanks D. Holm for informing him about e-MHD equations [Ho].

\section{REFERENCES}

[Br] Y. Brenier, Convergence of the Vlasov-Poisson system to the incompressible Euler equations, Comm. PDEs, 3-4:737-754, 2000.

[BrMaPu] Y. Brenier, N.J. Mauser, and M. Puel, Sur les limites quasineutres/non-relativistes: de Vlasov-Maxwell vers Euler incompressible, C. R. Acad. Sci., t 334, Srie I Math., 1-6, 2001.

[Da] C. Dafermos, Hyperbolic conservation laws in continuum physics, chapt. 5-2, Grundlehren der Mathematischen Wissenschaften, 325, Springer-Verlag, Berlin, 2000.

[DiLi] R.J. Di Perna and P.L. Lions, Global weak solutions of Vlasov-Maxwell systems, Comm. Pure and Appl. Math., 42:729-757, 1989.

[GISc] R. Glassey and J. Schaeffer, The relativistic Vlasov-Maxwell system in two space dimensions, Arch. Rational Mech. Anal., 141:331-354, 1998.

[Gr] E. Grenier, Boundary layers of $2 D$ inviscid fluids from a Hamiltonian viewpoint, Math. Res. Lett., 6:257-269, 1999.

[Ho] D.D. Holm, Notes on Electron MHD vs Euler-alpha, private communication to Y.B.

[KCY] A. Kingsep, K. Chukbar, and V. Yankov, Electron Magnetohydrodynamics, Review of Plasma Physics 16, ed. by B.B. Kodomtsev, New York, 1990.

[Li] P.L. Lions, Mathematical Topics in Fluid Mechanics, Vol. 1. Incompressible Models, Oxford Lecture in Mathematics and its Applications, Oxford Univ. Press, 1996.

[Pu1] M. Puel, Convergence of the Schrödinger Poisson system to the incompressible Euler equations, Comm. Partial Differential Equations, 27(11-12): 2311-2332, 2002.

[Pu2] M. Puel, Convergence of the Schrödinger Poisson system to the Euler equations under the influence of a large magnetic field, M2AN Math. Model Numer. Anal., 36(6): 1071-1090, 2002.

[Ya] H.T. Yau, Relative entropy and hydrodynamics of Ginzburg-Landau models, Lett. Math. Phys., 22(1):63-80, 1991. 